\title{
Impact of Left Atrial Volume Index on Discrepancy in Mitral Valve Area Assessed by Two-Dimensional Planimetry and Three-Dimensional Multi- Planar Reconstruction Technique
}

\author{
Mehrnoush Toufan ${ }^{1}$ and Zahra Jabbary ${ }^{1, *}$ \\ ${ }^{1}$ Cardiovascular Research Center, Shahid Madani Heart Center, Tabriz University of Medical Sciences, Tabriz, Iran \\ * Corresponding author: Zahra Jabbary, Cardiovascular Research Center, Shahid Madani Heart Center, Tabriz University of Medical \\ Sciences, Tabriz, Iran. Tel: +989144635732; Email: dr.z.jabbary@gmail.com
}

Received 2021 February 27; Revised 2021 February 28; Accepted 2021 August 17.

\begin{abstract}
Background: Mitral valve area (MVA) is technically measured using both two-dimensional (2D) planimetry and three-dimensional multiplanar reconstruction (3D-MPR) techniques; however, studies have always overestimated MVA using the former method.

Objectives: This study aimed to assess the correlation between MVA assessed by 2D and 3D techniques and the impact of left atrial volume index (LAVI) on the discrepancy between MVA assessed by two echocardiography techniques.

Methods: The data of 75 patients with moderate to severe mitral stenosis assessed by both 2D planimetry and 3D-MPR techniques were retrospectively reviewed. Clinical and echocardiographic variables were evaluated. Left atrial (LA) volume was determined using the biplane area-length method.

Results: The mean \pm SD MVA assessed by the 2D and 3D techniques was $1.03 \pm 0.24 \mathrm{~cm}^{2}$ and $0.99 \pm 0.25 \mathrm{~cm}^{2}$ with a mean discrepancy of $0.04 \pm 0.15 \mathrm{~cm}^{2}$, respectively. A strong association was observed between the MVA values assessed by 2D planimetry and 3D-MPR methods ( $\mathrm{r}$ coefficient $=0.817, \mathrm{P}<0.001$ ) indicating a slight discrepancy between the two techniques in assessing MVA. The pointed discrepancy was affected by none of the baseline characteristics and LAVI value. There was an adverse association between LAVI value and MVA measured by both $2 \mathrm{D}$ planimetry ( $\mathrm{r}$ coefficient $=-0.291, \mathrm{P}=0.011$ ) and 3D-MPR ( $\mathrm{r}$ coefficient $=-0.260, \mathrm{P}=0.024$ ) techniques.

Conclusion: In contrast to the left atrial dimension, the discrepancy in MVA values assessed by 2D planimetry and 3D-MPR techniques is not influenced by LAVI adjusted for baseline parameters.

Keywords: Indexplanimetry, Left atrial volume index, Mitral valve area
\end{abstract}

\section{Background}

Mitral valve area (MVA) is technically measured by two-dimensional (2D) planimetry with high precision to assess the severity of mitral pathological changes, such as stenosis (1). However, the improvement of imaging methods using three-dimensional techniques has been considered in some studies to achieve the accurate values of these changes and minimize diagnostic errors (especially in the presence of distorted valvular tip or poor echo widow) (2). The application of three-dimensional (3D) methods, such as real-time 3D echocardiography significantly reduced the possibility of mitral stenosis over-estimation due to accurate geometric assessment of valve dimensions and details of ischemic or rheumatic valvular changes with high resolution (3-4). Despite the development of 3D techniques, the 2D techniques are still used as common methods in many centers due to their availability and easier analysis. However, the technical limitations of 2D methods cause diagnostic challenges, especially in the accurate assessment of MVA (5). In other words, the reasons for the discrepancy between the two imaging techniques in the assessment of MVA, and the reply to the question "which patients would benefit from 3D as compared to 2D techniques for such assessment" have remained unknown.
In the early diastolic phase, a transient elevation in the pressure gradient across the left ventricle and left atrium (LA) can lead to a transmitral pressure gradient (6). This pressure gradient may be increased in the presence of mitral orifice obstruction (7). Moreover, the changes in left atrial (LA) dimensions, such as increased LA pressure are closely linked to mitral pathological changes, such as more severe mitral stenosis (8). Such changes can predispose the heart to arrhythmias, such as atrial fibrillation (AF), pulmonary congestion, pulmonary hypertension, progressing heart failure, and even death. In this regard, the left atrial volume index (LAVI) is now suggested as a prognostic factor to assess future cardiac events (9-10). This parameter is now recommended to assess LA size, LA remodeling, and diastolic functional status (11).

\section{Objectives}

Recent studies have reported that the MVA measurements obtained by 3D transesophageal echocardiography (3D-TEE) are significantly lower than the MVA measurements obtained by 2D planimetry in patients with mitral stenosis (MS), suggesting that the MVA measurements obtained by 2D planimetry may overestimate the true MVA. 
There have been few studies on the causes of discrepancy between the two measurements, and recognition of the patients that benefit from 3D-TEE for more accurate MVA measurements. Accordingly, this study hypothesized that there is a close association between LAVI and mitral pathological changes especially stenosis. The effect of LAVI on the discrepancy of MVA measurements using 2D planimetry and 3D multi-planar reconstruction (MPR) techniques was assessed for the first time in this study.

\section{Methods}

This is a retrospective study of 75 patients with moderate to severe mitral stenosis assessed by echocardiography. Those with moderate to severe mitral or aortic valve regurgitation, aortic stenosis, congenital heart defects, history of valvular heart interventions, pericardial disorders, or cardiac tumors were excluded from the study. The study protocol was approved by the Ethics Committee of Tabriz University of Medical Sciences, Tabriz, Iran.
The written informed consent was obtained from all participants after they were given adequate explanations regarding the objectives of the study. The baseline variables were recorded which included such information as the patients' demographic information, underlying cardiovascular risk profiles (hypertension, hyperlipidemia, current smoking status, diabetes mellitus, ischemic heart disorders, and chronic pulmonary disease), underlying rhythmic condition, and clinical functional status according to the New York Heart Association (NYHA) Classification. All patients underwent 2D transthoracic (2D-TTE) and transesophageal echocardiography (2D-TTE) on the same day. Initially, conventional 2D-TTE and then 2D-TEE was performed, followed by real-time three-dimensional transesophageal echocardiography (RT3D-TEE) image acquisition. The 2D image from the mitral valve was optimized at 70-90 degrees angles, where the best alignment occurred, and then the 3D image from the mitral valve was acquired in real-time, using 3D zoom and full volume modes (Figure 1).

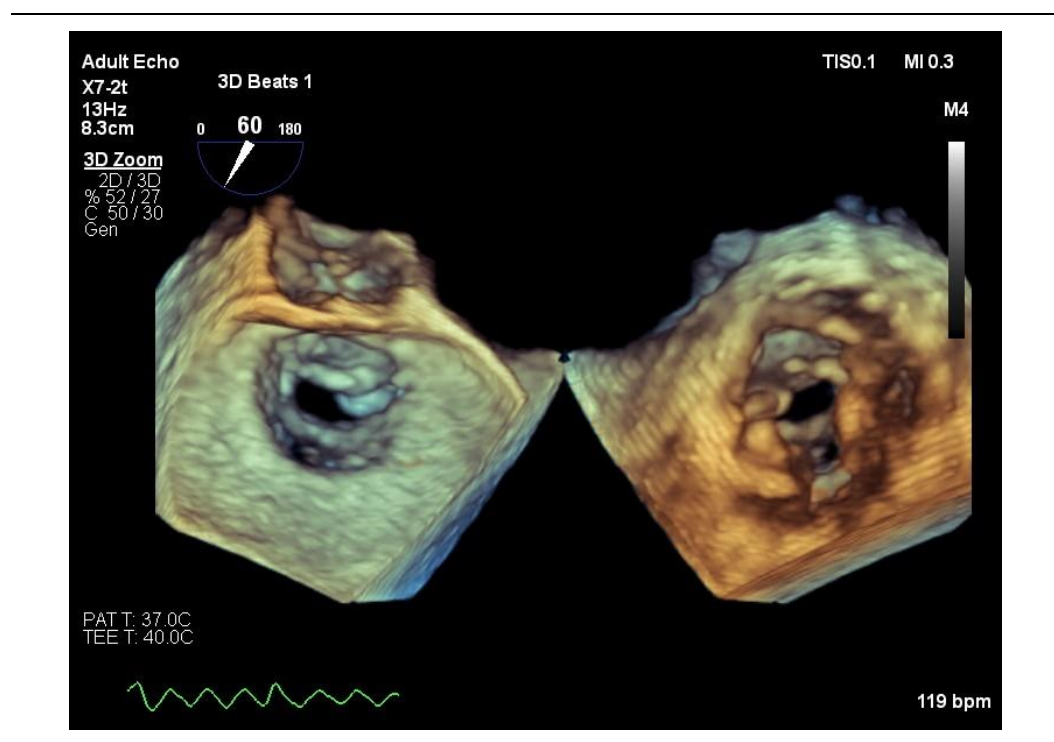

Figure 1. RT3D-TEE, rheumatic mitral valve stenosis (views from the left atrium and left ventricular sides, respectively from right to left)

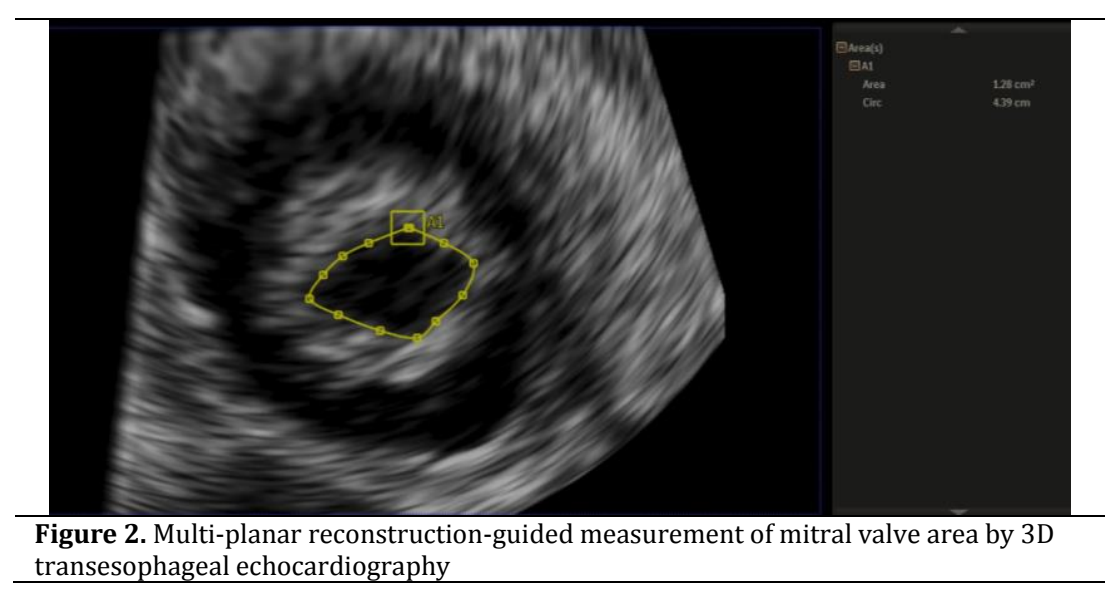




\begin{tabular}{lc}
\hline Table 1. Baseline characteristics of the study population \\
\hline Variables \\
\hline Age, mean years $\mathbf{\pm S D}$ & $53.61 \pm 12.06$ \\
Female gender, $\mathbf{n}(\%)$ & $53(70.7)$ \\
History of hypertension, $\mathbf{n}(\%)$ & $26(34.7)$ \\
History of diabetes mellitus, $\mathbf{n}(\%)$ & $3(4.0)$ \\
History of ischemic heart disease, $\mathbf{n}(\%)$ & $3(4.0)$ \\
History of smoking, $\mathbf{n}(\%)$ & $6(8.0)$ \\
History of cerebrovascular events, $\mathbf{n}(\%)$ & $1(1.3)$ \\
Cardiac rhythm, $\mathbf{n}(\%)$ & $34(45.3)$ \\
Normal sinus & $41(54.7)$ \\
Atrial fibrillation & $6(8.0)$ \\
NYHA classification, $\mathbf{n}(\%)$ & $51(68.0)$ \\
I & $3(4.0)$ \\
II & $15(20.0)$ \\
\hline III & \\
\hline
\end{tabular}

The details obtained from the transthoracic 2D planimetry and RT3D-TEE were described in detail. In this regard, MVA was assessed using both techniques. The normal value of MVA is in the range of 4-6 $\mathrm{cm}^{2}$; however, in the present study, all MVA values were less than or equal to $1.5 \mathrm{~cm}^{2}$. In the 3DMPR technique, the precise cross-sectional plane was located by the application of dedicated QLAB software (Philips Medical Systems) to cross the tips of the mitral valve, unaware of 2D echocardiography findings (Figure 2). In parallel, the MVA was measured by a 2D technique using an ultrasound system (GE Vivid S5). The MVA measurement was performed at the tip of the leaflets where maximal excursion of the leaflets was seen, in the parasternal short-axis view following the gain optimization and compression controls. The MVAs were measured for three different beats in patients with sinus rhythm and for five different beats in AF rhythm and averaged afterward. The techniques were performed by two echo-cardiologist completely blinded to each other's diagnosis. The LA volume was calculated using the biplane area-length method. Afterward, the LAVI was determined for the body surface area by the indexed LA volume and was categorized as normal $\left(\leq 28 \mathrm{~mL} / \mathrm{m}^{2}\right)$, mildly increased $\left(29-33 \mathrm{~mL} / \mathrm{m}^{2}\right)$, moderately increased $\left(34-39 \mathrm{~mL} / \mathrm{m}^{2}\right)$, and severely increased $\left(\geq 40 \mathrm{~mL} / \mathrm{m}^{2}\right)$. Moreover, left ventricular end-systolic and end-diastolic dimensions, as well as ejection fraction were measured. Tricuspid regurgitation velocity (continuous-wave Doppler) was measured for the calculation of pulmonary artery systolic pressure, using simplified Bernoulli equation, and right atrial pressure was determined based on the diameter of the inferior vena cava and its respiratory variability. Pulmonary artery systolic pressure was classified as normal $(<35 \mathrm{mmHg}$ ), mildly increased $(35-45 \mathrm{mmHg})$, moderately increased (45-60 $\mathrm{mmHg}$ ), and severely increased (>60 mmHg). Left atrial appendage (LAA) thrombus was defined as a solid mass with a clear margin in TEE that was visible throughout the cardiac cycle. The study endpoint was to assess the correlation between MVA assessed by the 2D and 3D techniques in addition to the impact of LAVI on the discrepancy between the two values of MVA determined by two echocardiography techniques.

The quantitative and qualitative (or categorical) variables in the statistical analysis were presented as mean \pm standard deviation (SD) and frequency (percentage), respectively. The Pearson's or Spearman's correlation tests were used to assess the correlation between the quantitative variables. The $\mathrm{p}$ values less than 0.05 were considered statistically significant. The data were analyzed using the SPSS software (version 23.0; IBM, Armonk, New York).

\section{Results}

The mean $\pm S D$ age of patients in this study was $53.61 \pm 12.06$ years (the age range of 26-81 years). Most patients $(70.7 \%)$ in this study were female. Based on cardiovascular risk profiles (Table 1), hypertension was the most common risk factor observed in about one-third of patients. About half of the participants suffered from atrial fibrillation. In addition, one-fourth of patients suffered from significant functional dysfunction indicated by NYHA classes III and IV.

The echocardiography findings obtained by the 2D planimetry and 3D-MPR techniques are presented in Table 2. The mean \pm SD MVA values assessed by the two mentioned techniques were $1.03 \pm 0.24 \mathrm{~cm}^{2}$ and $0.99 \pm 0.25 \mathrm{~cm}^{2}$, respectively with a mean discrepancy of $0.04 \pm 0.15 \mathrm{~cm}^{2}$. A strong association was revealed between the MVA values assessed by 2D planimetry and 3D-MPR methods ( $\mathrm{r}$ coefficient $=0.817, \mathrm{P}<0.001$ ) indicating a slight discrepancy between the two techniques in assessing MVA. According to the multivariable linear regression modeling (Table 3), the discrepancy was affected by none of the baseline characteristics. In this regard, the discrepancy was not also impacted by the LAVI value.

The mean \pm SD LAVI value was also $72.97 \pm 27.54$ $\mathrm{mL} / \mathrm{m}^{2}$, indicating a moderate increase of $5.3 \%$ and a severe increase of $94.7 \%$. There was an adverse association between LAVI and MVA values measured by 2D planimetry ( $\mathrm{r}$ coefficient $=-0.291$, 


\begin{tabular}{|c|c|}
\hline Variables & \\
\hline MVA assessed by 2D technique, mean \pm SD & $1.03 \pm 0.24$ \\
\hline MVA assessed by $3 D$ technique, mean $\pm S D$ & $0.99 \pm 0.25$ \\
\hline LAVI value, mean \pm SD & $72.97 \pm 27.54$ \\
\hline Reduced LVEF ( $\leq 45 \%)$, n (\%) & $11(14.7)$ \\
\hline Thrombus in left atrial appendage, n (\%) & $12(16.0)$ \\
\hline Pulmonary hypertension, n (\%) & $\mathrm{N}(\%)$ \\
\hline None & $20(26.7)$ \\
\hline Mild & $22(29.3)$ \\
\hline Moderate & $16(21.3)$ \\
\hline Severe & $17(22.7)$ \\
\hline
\end{tabular}

Table 3. Multivariable linear regression analysis for determining the effect of baseline variables on the discrepancy between MVA assessed by $2 \mathrm{D}$ and $3 \mathrm{D}$ methods

\begin{tabular}{|c|c|c|c|c|}
\hline \multirow{2}{*}{ Variable } & \multicolumn{2}{|c|}{ Unstandardized Coefficients } & \multirow[b]{2}{*}{$\mathbf{T}$} & \multirow[b]{2}{*}{ P-value } \\
\hline & B & Std. Error & & \\
\hline Age & -0.002 & 0.002 & -0.982 & 0.330 \\
\hline Gender & 0.001 & 0.041 & 0.016 & 0.987 \\
\hline HTN & 0.052 & 0.045 & 1.165 & 0.248 \\
\hline DM & 0.082 & 0.099 & 0.830 & 0.410 \\
\hline CS & 0.093 & 0.070 & 1.327 & 0.189 \\
\hline IHD & -0.019 & 0.099 & -0.192 & 0.848 \\
\hline CVA & -0.068 & 0.164 & -0.412 & 0.682 \\
\hline Rhythm & 0.030 & 0.046 & 0.655 & 0.515 \\
\hline NYHA & 0.006 & 0.022 & 0.289 & 0.774 \\
\hline LAVI value & 7.015 & 0.001 & 0.087 & 0.931 \\
\hline
\end{tabular}

$\mathrm{P}=0.011)$ and 3D-MPR ( $\mathrm{r}$ coefficient $=-0.260$, $\mathrm{P}=0.024$ ) techniques (Figures 3 ).

Most of the patients in this study suffered from pulmonary hypertension (PH). In total, $21.3 \%$ and $22.7 \%$ of patients suffered from moderate and severe
$\mathrm{PH}$, respectively. The mean LAVI was strongly associated with the severity of $\mathrm{PH}$, so that the mean \pm SD LAVI in mild, moderate, and severe $\mathrm{PH}$ was determined at $62.55 \pm 16.90,87.78 \pm 40.59$, and 81.95 \pm 24.77 , respectively ( $\mathrm{P}=0.008)$.

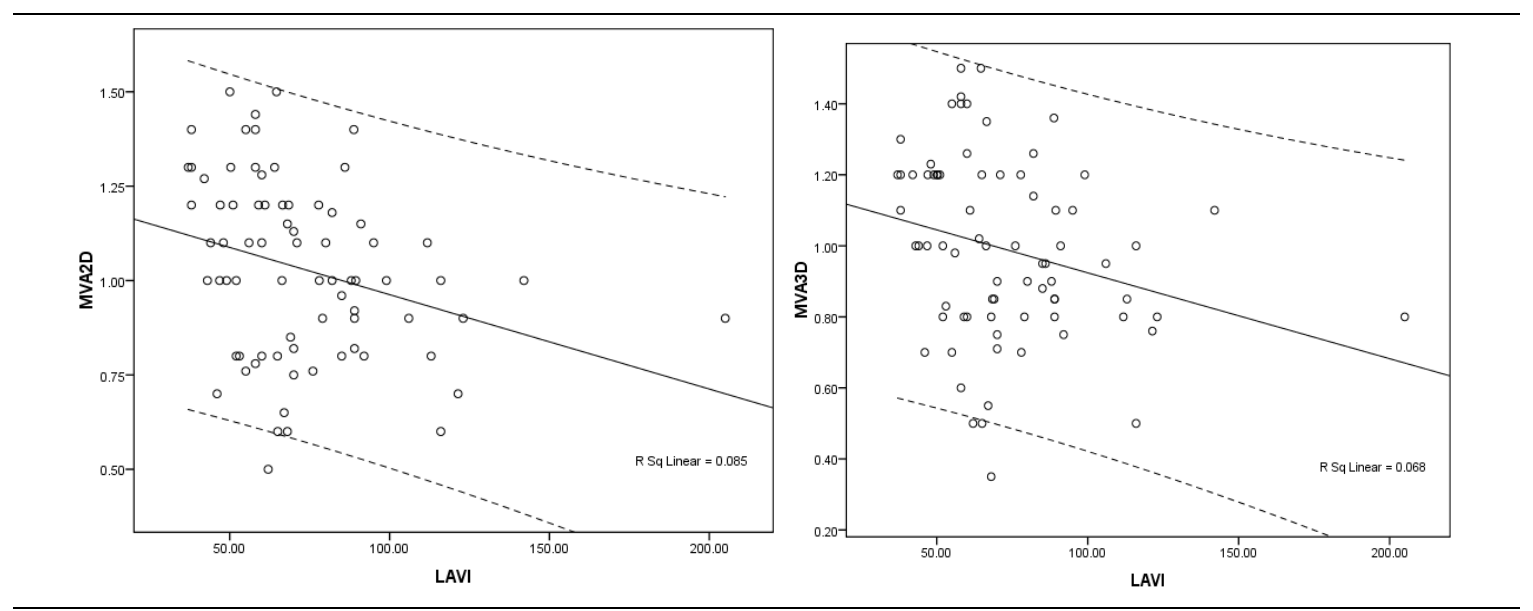

Figure 3. The association between LAVI value and MVA assessed by 2D planimetry and 3D-MPR

\section{Discussion}

The LA dimensions are directly related to the indicators of valvular function, especially mitral valve, as well as pulmonary pressures. In addition, the cross-sectional MVA represents the severity of mitral valve stenosis. Therefore, it seems that the MVA is mainly affected by dimensional changes in the left atrium. Based on the existing studies, both parameters play an important prognostic role in predicting clinical outcomes in patients with mitral valve involvement. Therefore, accurate evaluation of these parameters is essential for the patient's survival as well as selecting the optimal therapeutic interventions for valvular diseases. Today, both 2D and 3D imaging tools have been used to evaluate valvular parameters, such as valvular pathological changes, even though each tool has its advantages and limitations. Although 2D methods are more available and far more cost-effective compared to the 3D methods, these are associated with the overestimation of some apparatus parameters in 
certain views. In addition, despite the much higher accuracy of 3D imaging tools, these tools are less available, and working with these tools and interpreting findings requires a much more experienced operator. Due to the increasing use of both instruments in diagnostic centers, the assessment of the compatibility of the results of the two instruments is necessary for the evaluation of heart valve disorders. Second, it should be noted that these assessments may be influenced by other cardiovascular dimensions as well.

Regarding the important findings of the present study, one can refer to the fact that although the use of the 2D method was associated with a slight overestimation in the assessment of the MVA, this diagnostic error was very insignificant and the agreement between the 2D and 3D diagnostic tools was high and acceptable. In addition, there was a strong inverse correlation between the LAVI and the MVA. In other words, changes in the MVA were essentially accompanied by changes in the other index. Therefore, based on physiological principles, the LAVI value can be naturally affected by changes in MVA. Another important point about the diagnostic discrepancy between the two diagnostic tools was that this diagnostic discrepancy between the two methods is not affected by demographic characteristics of the patients or cardiovascular indices as well as the value of left atrial volume parameters. Therefore, it seems that this discrepancy may only be influenced by the operator's experience in performing and interpreting the results.

The results of a study conducted by Zamorano et al. on patients with rheumatic mitral valve stenosis demonstrated that MVA measured by 3D-TTE was closer to the corresponding values measured by invasive evaluation approaches, compared to conventional 2D echo-Doppler. They noted that real-time three-dimensional echocardiography (RT3DE) could improve the evaluation of MS severity in patients with conflicting results due to the application of different methods (12). In addition, Schlosshan et al. reported that MVA measured by $3 \mathrm{D}$-TEE had the best correlation and agreement with MVA obtained by continuity equation (CE), and was significantly lower than that acquired by $2 \mathrm{D}$ planimetry and pressure half time (PHT) method (13). The study performed by Chu et al. included patients with calcific mitral stenosis and reported that there was a good agreement between the value of MVA determined by RT3DE and $C E$, rather than the value of MVA obtained by PHT. They reported that RT3DE provided an accurate measurement of MVA in patients with calcific mitral stenosis (14). The results of these studies were in line with the results of the present study.

In a similar study carried out by Min et al. in 2013 (15), the MVA measured by 2D planimetry had high agreement with 3D methods; however, 2D planimetry overestimated MVA by $0.19 \mathrm{~cm}^{2}$. In contrast to the observation in the present study, LA dimensions were independent determinants of the discrepancy in MVA measurements assessed by the two diagnostic tools. Based on the findings, 3D-MPR imaging can accurately localize the mitral valve tip. The values of MVA assessed by the 3D method had been usually smaller than those measured by 2D methods; therefore, the application of 2D techniques might not lead to accurate measurements of MVA (12). This limitation is related to the lack of an ideal 2D shortaxis plane crossing the tips of the mitral leaflets (13). In other words, the imaging plane should cross the tips of both leaflets to obtain an accurate value of MVA by 2D planimetry, which is no easy task to perform.

Regarding the evaluation of the relationship between the MVA and LAVI, it has been demonstrated that the LAVI was mainly affected by the presence of both dimensional and functional parameters, such as the severity of mitral stenosis, the presence of atrial fibrillation, and left ventricular mass index (16). Moreover, there was a strong inverse correlation between the LAVI and the MVA (as demonstrated in this study and those of others). Therefore, it seems that the evaluation of both indicators will be very useful in the prognostic evaluation of patients, especially when the evaluations are performed using a two-dimensional technique and there is a possibility of estimation error. Moreover, the value of each of these two indicators may be influenced by some underlying indicators that should be considered as confounding factors. Eventually, the evaluation of the correlation between the two indicators should also be considered in the absence of confounding factors in future studies, which are recommended to be conducted with larger sample size.

\section{Conclusion}

Based on the obtained results, there is a high agreement between the two methods in assessing the mitral surface and the results are reliable accordingly. In contrast to the left atrial dimension, the discrepancy between the two measurements of MVA conducted by 2D or 3D techniques is not affected by the LAVI. However, this discrepancy may be minimized through the improvement of the operator's experience in using the 2D technique. It seems that more studies with a larger sample size are needed to evaluate the causes of discrepancy between the two measurements and recognize the patients who would benefit from threedimensional echocardiography for more accurate MVA measurements.

\section{Acknowledgments}

None. 


\section{Footnotes}

Conflicts of Interest: The authored declare that they have no conflict of interest regarding the publication of the present study.

\section{Funding/ Support: None.}

\section{References}

1. Sadeghian H, Rezvanfard M, Jalali A. Measurement of mitral valve area in patients with mitral stenosis by $3 \mathrm{D}$ echocardiography: A comparison between direct planimetry on 3D zoom and 3D quantification. Echocardiography. 2019;36(8):1509-14. doi: 10.1111/echo.14397. [PubMed: 31287584].

2. Uygur B, Celik O, Ustabasioglu FE, Akinci O, Erturk M. Threedimensional transesophageal echocardiography vs cardiac magnetic resonance in the assessment of planimetric mitral valve area in rheumatic mitral stenosis. Echocardiography. 2018;35(10):1621-5. doi: 10.1111/echo.14096. [PubMed: 29981197].

3. Toufan M, Aghdam NK. The most accurate method for measurement of mitral valve area in mitral stenosis by direct planimetery three dimensional transesophageal echocardiography (3D TEE). Adv Biosc Clin Med. 2018;6(4):33. doi: $10.7575 /$ aiac.abcmed.v.6n.4p.33.

4. Wu VC, Takeuchi M. Three-dimensional echocardiography: current status and real-life applications. Acta Cardiol Sin. 2017;33(2):107-18. doi: 10.6515/acs20160818a. [PubMed: 28344414]

5. Mohamed AA, Omran A, Hussein MA. Assessment of mitral valve area by $3 \mathrm{D}$ echocardi-ography in rheumatic mitral stenosis: Validation of offline 3D planimetry measure. J Saudi Heart Assoc. 2013;25:113-72. doi: 10.1016/j.jsha.2013.03.047.

6. Del Rio JM, Grecu L, Nicoara A. Right ventricular function in left heart disease. Semin Cardiothorac Vasc Anesth. 2019;23(1):88107. doi: $10.1177 / 1089253218799345$.

7. El Sabbagh A, Reddy YNV, Barros-Gomes S, Borlaug BA, Miranda WR, Pislaru SV, et al. Low-gradient severe mitral stenosis: hemodynamic profiles, clinical characteristics, and outcomes. I Am Heart Assoc. 2019;8(5):e010736. doi: 10.1161/JAHA.118.010736. [PubMed: 30793648].
8. Abhayaratna WP, Seward JB, Appleton CP, Douglas PS, Oh JK, Tajik AJ, et al. Left atrial size: physiologic determinants and clinical applications. J Am Coll Cardiol. 2006;47(12):2357-63. doi: 10.1016/j.jacc.2006.02.048. [PubMed: 16781359].

9. Takeuchi M, Kitano T, Nabeshima Y, Otsuji Y, Otani K. Left ventricular and left atrial volume ratio assessed by threedimensional echocardiography: Novel indices for evaluating age-related change in left heart chamber size. Physiol Rep. 2019;7(23):e14300. doi: 10.14814/phy2.14300. [PubMed: 31814325].

10. Cho IJ, Jeong H, Choi JY, Lee SE, Chang HJ. Prognostic Implications of the left atrial volume index in patients with progressive mitral stenosis. $J$ Cardiovasc Imaging. 2019;27(2):122-33. doi: 10.4250/jcvi.2019.27.e20. [PubMed: 30993947].

11. Bangalore S, Yao SS, Chaudhry FA. Role of left atrial size in risk stratification and prognosis of patients undergoing stress echocardiography. J Am Coll Cardiol. 2007;50(13):1254-62. doi: 10.1016/j.jacc.2007.06.025. [PubMed: 17888843].

12. Zamorano J, Cordeiro P, Sugeng L, Perez de Isla L, Weinert L, Macaya C, et al. Real-time three-dimensional echocardiography for rheumatic mitral valve stenosis evaluation. An accurate and novel approach. J Am Coll Cardiol. 2004;43(11):2091-6. doi: 10.1016/j.jacc.2004.01.046. [PubMed: 15172418].

13. Schlosshan D, Aggarwal G, Mathur G, Allan R, Cranney G. Realtime 3D transesophageal echocardiography for the evaluation of rheumatic mitral stenosis. JACC Cardiovasc Imaging 2011;4(6):580-8. doi: 10.1016/j.jcmg.2010.12.009. [PubMed: 21679891]

14. Chu JW, Levine RA, Chua S, Poh KK, Morris E, Hua L, et al. Assessing mitral valve area and orifice geometry in calcific mitral stenosis: a new solution by real-time three-dimensional echocardiography. J Am Soc Echocardiogr. 2008;21(9):1006-9. doi: 10.1016/j.echo.2008.05.010. [PubMed: 18620839].

15. Min SY, Song JM, Kim YJ, Park HK, Seo MO, Lee MS, et al. Discrepancy between mitral valve areas measured by two-dimensional planimetry and three-dimensional transoesophageal echocardiography in patients with mitral stenosis. Heart. 2013;99(4):253-8. doi: 10.1136/heartjnl2012-302742. [PubMed: 23125249].

16. Cho IJ, Jeong H, Choi JY, Lee SE, Chang HJ. Prognostic implications of the left atrial volume index in patients with progressive mitral stenosis. J Cardiovasc Imaging. 2019;27(2):122-33. doi: 10.4250/jcvi.2019.27.e20. [PubMed: 30993947]. 